\title{
Stathmin-2: adding another piece to the puzzle of TDP-43 proteinopathies and neurodegeneration
}

\author{
Jonathan D. Glass \\ Departments of Neurology and Pathology, Emory University School of Medicine, Atlanta, Georgia, USA.
}

\begin{abstract}
Cytoplasmic aggregated proteins are a common neuropathological feature of neurodegenerative diseases. Cytoplasmic mislocalization and aggregation of TAR-DNA binding protein 43 (TDP-43) is found in the majority of patients with amyotrophic lateral sclerosis (ALS) and in approximately $50 \%$ of patients dying of frontotemporal lobar degeneration (FTLD). In this issue of the $\mathrm{JCl}$, Prudencio, Humphrey, Pickles, and colleagues investigated the relationship of TDP-43 pathology with the loss of stathmin-2 (STMN2), an essential protein for axonal growth and maintenance. Comparing genetic, cellular, and neuropathological data from patients with TDP-43 proteinopathies (ALS, ALS-frontotemporal dementia [ALS-FTD], and FTLD-TDP-43 [FTLD-TDP]) with data from patients with non-TDP-related neurodegenerations, they demonstrate a direct relationship between TDP-43 pathology and STMN2 reduction. Loss of the normal transcription suppressor function of TDP-43 allowed transcription of an early termination cryptic axon, resulting in truncated, nonfunctional mRNA. The authors suggest that measurement of truncated STMN2 mRNA could be a biomarker for discerning TDP proteinopathies from other pathologies.
\end{abstract}

\section{Cytoplasmic aggregated proteins in neurodegenerative disorders}

A common neuropathological feature of several neurodegenerative diseases is the mislocalization and aggregation of Tar DNA-binding protein 43 (TDP-43), which is a member of the family of heterogenous nuclear ribonucleoproteins (hnRNPs) and is normally ubiquitously expressed in nuclei throughout the CNS (1). Intensive investigations regarding the normal function of TDP-43 and its role in the pathogenesis of neurodegeneration have identified a variety of pathways in which TDP-43 perturbations lead to neuronal toxicity. Originally identified as the major component of ubiquitinated cytoplasmic inclusions in amyotrophic lateral sclerosis (ALS) and frontotemporal lobar degeneration (FTLD) (2), TDP-43 pathology is now recognized as a neuropathological feature of a substantial proportion of cases of Alzheimer's disease as well as Parkinson's and Huntington's diseases, and even the degenerative muscle disease inclusion body myopathy.

The connection between TDP-43 and neurodegeneration was sealed by the discovery of gene mutations in its parent gene, $T A R D P B$, that are found in rare familial cases of ALS and FTLD, all of which demonstrate the pathognomonic finding of aggregated neuronal cytoplasmic TDP43 inclusions. Given this neuropathology, it seems apparent that the mechanism of TDP-43-associated toxicity likely relates to cytoplasmic TDP-43 aggregates or the loss of TDP- 43 protein from the nucleus,

Related Article: p. 6080

Conflict of interest: The author receives funding for clinical trials from Biogen and Genentech.

or a combination of both. The pathways identified as being related to this pathology are now numerous.

Cytoplasmic aggregated proteins are a common feature of most neurodegenerative diseases, and there is some debate about whether these inclusions are directly toxic to neurons (3), or are possibly protective, acting to sequester toxic soluble oligomers (4-6). Indeed, TDP-43 cytoplasmic aggregates are seen in the majority of patients dying of sporadic or familial ALS and approximately $50 \%$ of patients with FTLD. However, a number of TDP-43 cellular and animal models demonstrate that TDP-43 mislocalization and aggregation are not required for neurotoxicity (7-9), bringing into question the role of mislocalization and aggregation in disease pathogenesis. Though phosphorylated TDP-43 (p-TDP-43) inclusions are necessary for the neuropathological diagnosis of ALS and FTLD with TDP-43 pathology (FTLD-TDP), the proportion of cells showing this pathological feature, even at end stage, may be minor. These minor quantities contrast with the overwhelming burden of amyloid $\beta$ and p-tau that is seen in most patients dying of Alzheimer's disease. This contradistinction certainly does not discount the importance of TDP-43 in ALS and FTLD, but may suggest (as do the animal models) that what we see under the microscope may not tell the whole story.

A particularly informative discovery regarding TDP-43-related neurodegeneration is its interaction with the RNA-binding protein ataxin-2 (ATX2). ATX2 is a regulator of stress granule assembly, and there is evidence that sequestered TDP- 43 in stress granules leads to the formation of insoluble TDP-43 aggregates (10). Remarkably, reducing or deleting ATX2 in transgenic TDP-43 mice substantially reduces TDP43 aggregate pathology and results in an extraordinary improvement in survival (11).

TDP-43 is also a transcription suppressor. In the absence of TDP-43, i.e., when TDP-43 is cleared from the nucleus 


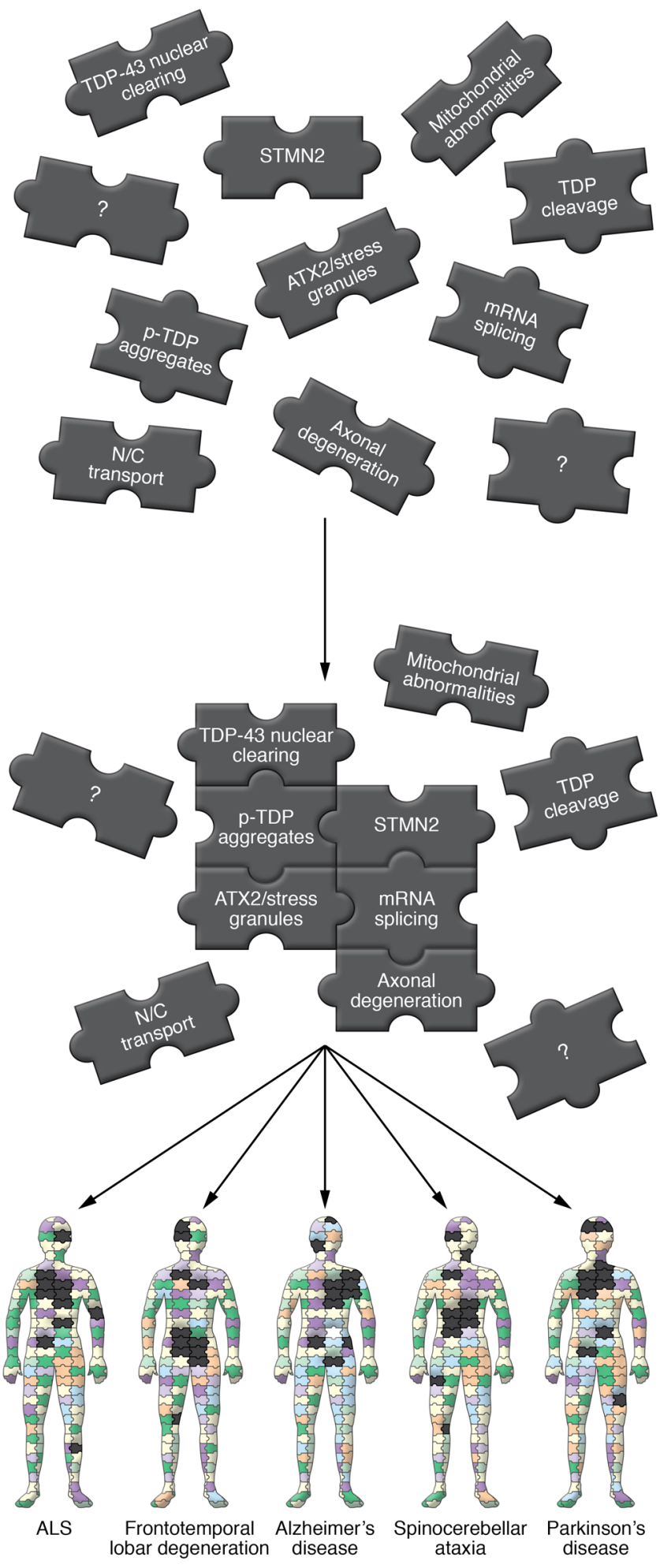

Figure 1. The puzzle of neurodegeneration. Multiple processes and pathways leading to neurodegeneration are identified from experimental models and human neuropathology. Some of those pathways, proteins, and genes may fit together as an organized model of disease. STMN2 is shown as a central component of a pathway that includes TDP-43 proteinopathy - i.e., TDP-43 nuclear clearing, formation of phosphorylated cytoplasmic aggregates, and reduction of its normal transcription suppressor function in STMN2 mRNA. It is unclear how other pieces fit in, and still others remain missing. These same pathways are likely common to several neurodegenerative diseases. $\mathrm{N} / \mathrm{C}$ transport, nucleocytoplasmic transport. as it mislocalizes to the cytoplasm, cryptic exons are exposed and transcribed, creating aberrant mRNAs that lead to neurotoxicity in ALS models (12-14). Recently, this TDP-43 suppressor function was specifically implicated in suppressing a cryptic early polyadenylation site in the pre-mRNA for the protein stathmin-2 (STMN2). Loss of TDP-43 allows incorporation of a premature poly A tail, resulting in a truncated and nonfunctional form of STMN2 $(15,16)$. STMN2 is a highly conserved cytosolic protein essential for axonal outgrowth and maintenance. STMN2-knockout mice develop late-onset, predominantly motor axonopathy (17) paralleling that seen in human ALS.

\section{Truncated STMN2}

In this issue of the JCI, Prudencio, Humphrey, Pickles, and colleagues (18) provide further convincing evidence that TDP-43 pathology is associated with the appearance of the nonfunctional, truncated STMN2 as well as a reduction of the normal long form of STMN2. The researchers focused on whether the presence of truncated STMN2 could be used as a biomarker for differentiating the underlying pathology in patients with FTLD. Only half of the patients with frontotemporal dementia (FTD) showed TDP-43 pathology (FTLD-TDP) at autopsy. The majority of those without TDP-43 pathology had pathological abnormalities of the microtubule-binding protein tau (FTLDtau). Clinically, these entities are indistinguishable. Given that the mechanisms of disease are likely distinct, differentiating FTLD-TDP from FTLD-tau during life will be important for the testing of therapeutics in clinical trials.

Prudencio, Humphrey, Pickles, and colleagues (18) sought to distinguish patients with FTLD-TDP from those with FTLDtau by comparing genetic, cellular, and neuropathological data from patients with TDP-43 proteinopathies (ALS, ALS-FTD, FTLD-TDP) with data from those with nonTDP-related neurodegenerations (SOD1-related ALS, FTLD-tau, progressive supranuclear palsy) and from control subjects. The authors interrogated brain and spinal cord tissues from these patient groups by RNA-Seq and found a consistent correlation between the presence of the truncated STMN2 transcript, the reduction of fulllength STMN2 RNA, and the presence of 
TDP-43 pathology. Quantitative immunoassay measurement of $\mathrm{p}-\mathrm{TDP}-43$ protein, which is the pathological form found in mislocalized cytoplasmic TDP-43 aggregates, also correlated directly with RNA levels of truncated STMN2. Interestingly, and perhaps not surprisingly, truncated STMN2 RNA was found only in brains from patients with TDP-43 proteinopathy. Truncated STMN2 RNA was concentrated in brain regions with a high burden of TDP-43 pathology (frontal and temporal cortex in FTLD; motor cortex and spinal cord in ALS) and was negligible in regions without TDP-43 pathology (cerebellum). Spinal cord tissue from patients with SOD1 ALS, the relatively rare exception to the presence of pathological TDP-43 inclusions in ALS, also showed no evidence of truncated STMN2 (18).

Further experiments using induced pluripotent stem cell (iPSC-derived) motor neurons confirmed that depletion of nuclear TDP-43 unmasked the STMN2 cryptic exon and the appearance of truncated RNA. These neurons were depleted of nuclear TDP-43 but failed to develop p-TDP-43 cytoplasmic inclusions, confirming the assumption that it is the loss of TDP-43 and not the mislocalization that drives the STMN2 changes. Also, iPSC-derived motor neurons generated from patients with pathogenic TARDBP mutations that (perhaps surprisingly) did not show reduced nuclear TDP-43, did not generate the truncated STMN2 RNA (18). These data are important, as they confirm the relationship between the reduction of full-length and the presence of truncated STMN2 RNA in TDP-43-related neurodegeneration. As suggested by the authors, these data represent an essential step toward the possibility of measuring truncated STMN2, perhaps from cerebrospinal fluid (CSF), as a biomarker for TDP-43 proteinopathy. A clinical assay for truncated STMN2 would not only allow in vivo differentiation of FTLD-TDP from FTLDtau, but could also establish an indicator for TDP-43 pathology in other neurodegenerative diseases.

\section{Fitting multiple genes, proteins, and pathways into a model}

Prudencio, Humphrey, Pickles, and colleagues focused on the loss of full-length STMN2 as a consequence of the unmask- ing of the early polyadenylation site due to the mislocalization and loss of TDP43 (18). However, previous data from patients' fibroblasts and fibroblast-derived neurons (iNeurons) carrying pathogenic TARDBP mutations demonstrated reduced STMN2 RNA in the absence of TDP-43 mislocalization (16). Similarly, mice carrying ALS-causing human TARDBP mutations show abnormal RNA splicing without TDP-43 mislocalization or aggregation (19). Thus, nuclear clearing of TDP-43 and/or sequestering of TDP-43 into cytoplasmic inclusion bodies may be sufficient, but not absolutely necessary, for exposure of the cryptic exon, truncation of STMN2 RNA, and, ultimately, the reduction of STMN2 protein. An interesting finding in the study by Prudencio, Humphrey, Pickles, and colleagues is that brain tissue from patients with FTLD-tau showed reductions in full-length STMN2 mRNA similar to those see in patients with FTLD-TDP, but in the absence of truncated STMN2 (18). Though unexplained, this reduction specific to full-length STMN2 raises the possibility that loss of STMN2, with or without TDP-43 pathology, may represent a final common pathway of neurodegeneration.

The interaction between TDP-43 and STMN2 in ALS and FTLD highlights the importance of axonal biology as a possible driver of disease (20). STMN2 is enriched in motor neurons, and its presence is essential for axonal growth and maintenance $(16,21)$. ALS animal models and clinical and pathological observations in human ALS show axonal degeneration to be an early feature of disease that is separate from the loss of neuronal cell bodies (2224). We also know from studies in the slow Wallerian degeneration (WldS) mouse and the discovery of the SARM1 axonal degeneration regulatory gene that the death of axons and neuronal cell bodies is driven by distinct underlying mechanisms $(25,26)$. In ALS animal models, in which therapeutic intervention completely protects motor neuron cell bodies, axons still degenerate and animals die in typical fashion $(27,28)$.

The challenge for deciphering the causes and drivers of ALS, FTLD, and other neurodegenerative diseases is how to fit the various clinical and experimental observations involving multiple genes, proteins, and pathways into a cohesive model of disease pathogenesis. The multiple functions and dysfunctions of TDP43, including its newly recognized influence on axonal growth and maintenance, are prime examples of the mechanistic complexities that underlie age-related dysfunction and degeneration of the nervous system. It is unlikely that the varied phenotypes of the broken nervous system represent distinct and unrelated pathological pathways. This puzzle has many pieces (Figure 1). STMN2 may be a centerpiece that connects several of those pieces together.

\section{Acknowledgments}

Thank you to the patients and families who donate tissues for research, without which this type of research cannot be accomplished. The author received research funding from the National Institute of Neurological Disorders and Stroke (NINDS), NIH; the Muscular Dystrophy Association, the ALS Association; and ALS Finding a Cure.

Address correspondence to: Jonathan D. Glass, Departments of Neurology and Pathology, Emory University School of Medicine, 101 Woodruff Circle Suite 6117, Atlanta, Georgia 30322, USA. Phone: 404.727.3507; Email:jglas03@emory.edu.

1. Kwong LK, Neumann M, Sampathu DM, Lee VM, Trojanowski JQ. TDP-43 proteinopathy: the neuropathology underlying major forms of sporadic and familial frontotemporal lobar degeneration and motor neuron disease. Acta Neuropathol. 2007;114(1):63-70.

2. Neumann M, et al. Ubiquitinated TDP43 in frontotemporal lobar degeneration and amyotrophic lateral sclerosis. Science. 2006;314(5796):130-133.

3. Johnson BS, Snead D, Lee JJ, McCaffery JM, Shorter J, Gitler AD. TDP-43 is intrinsically aggregation-prone, and amyotrophic lateral sclerosis-linked mutations accelerate aggregation and increase toxicity. J Biol Chem. 2009;284(30):20329-20339.

4. Bodner RA, et al. Pharmacological promotion of inclusion formation: a therapeutic approach for Huntington's and Parkinson's diseases. Proc Natl Acad Sci U S A. 2006;103(11):4246-4251.

5. Brotherton TE, Li Y, Glass JD. Cellular toxicity of mutant SOD1 protein is linked to an easily soluble, non-aggregated form in vitro. Neurobiol Dis. 2013;49:49-56.

6. Witan H, Kern A, Koziollek-Drechsler I, Wade R, Behl C, Clement AM. Heterodimer formation of wild-type and amyotrophic lateral sclerosis-causing mutant $\mathrm{Cu} / \mathrm{Zn}$-superoxide dismutase induces toxicity independent of protein aggregation. Hum Mol Genet. 2008;17(10):1373-1385.

7. Gendron TF, Petrucelli L. Rodent models of 
TDP-43 proteinopathy: investigating the mechanisms of TDP-43-mediated neurodegeneration. J Mol Neurosci. 2011;45(3):486-499.

8. Igaz LM, et al. Dysregulation of the ALS-associated gene TDP-43 leads to neuronal death and degeneration in mice. JClin Invest. 2011;121(2):726-738

9. Wegorzewska I, Baloh RH. TDP-43-based animal models of neurodegeneration: new insights into ALS pathology and pathophysiology. Neurodegener Dis. 2011;8(4):262-274.

10. Li YR, King OD, Shorter J, Gitler AD. Stress granules as crucibles of ALS pathogenesis. J Cell Biol. 2013;201(3):361-372.

11. Becker LA, et al. Therapeutic reduction of ataxin-2 extends lifespan and reduces pathology in TDP-43 mice. Nature. 2017;544(7650):367-371.

12. Buratti E, Brindisi A, Pagani F, Baralle FE. Nuclear factor TDP-43 binds to the polymorphic TG repeats in CFTR intron 8 and causes skipping of exon 9: a functional link with disease penetrance. Am J Hum Genet. 2004;74(6):1322-1325.

13. Donde A, et al. Splicing repression is a major function of TDP-43 in motor neurons. Acta Neuropathol. 2019;138(5):813-826.

14. Ling JP, Pletnikova O, Troncoso JC, Wong PC. TDP- 43 repression of nonconserved cryptic exons is compromised in ALS-FTD. Science.
2015;349(6248):650-655

15. Klim JR, et al. ALS-implicated protein TDP43 sustains levels of STMN2, a mediator of motor neuron growth and repair. Nat Neurosci. 2019;22(2):167-179.

16. Melamed Z, et al. Premature polyadenylation-mediated loss of stathmin-2 is a hallmark of TDP-43-dependent neurodegeneration. Nat Neurosci. 2019;22(2):180-190.

17. Liedtke W, Leman EE, Fyffe RE, Raine CS, Schubart UK. Stathmin-deficient mice develop an age-dependent axonopathy of the central and peripheral nervous systems. Am J Pathol. 2002;160(2):469-480.

18. Prudencio M, et al. Truncated stathmin- 2 is a marker of TDP-43 pathology in frontotemporal dementia. JClin Invest. 2020;130(11):6080-6092.

19. Arnold ES, et al. ALS-linked TDP-43 mutations produce aberrant RNA splicing and adult-onset motor neuron disease without aggregation or loss of nuclear TDP-43. Proc Natl Acad Sci U S A 2013;110(8):E736-E745.

20. Suzuki N, Akiyama T, Warita H, Aoki M. Omics approach to axonal dysfunction of motor neurons in amyotrophic lateral sclerosis (ALS). Front Neurosci. 2020;14:194.

21. Duncan JE, Lytle NK, Zuniga A, Goldstein LS. The microtubule regulatory protein stathmin is required to maintain the integrity of axonal microtubules in Drosophila. PLOS ONE. 2013;8(6):e68324.

22. Dadon-Nachum M, Melamed E, Offen D. The "dying-back" phenomenon of motor neurons in ALS. J Mol Neurosci. 2011;43(3):470-477.

23. Fischer LR, et al. Amyotrophic lateral sclerosis is a distal axonopathy: evidence in mice and man. Exp Neurol. 2004;185(2):232-240.

24. Fischer-Hayes LR, Brotherton T, Glass JD. Axonal degeneration in the peripheral nervous system: implications for the pathogenesis of amyotrophic lateral sclerosis. Exp Neurol. 2013;246:6-13.

25. Glass JD. The ultimate question: why do axons degenerate? A tribute to the work and mentorship of John W. Griffin, MD. JPeripher Nerv Syst. 2012;17 Suppl 3:24-29.

26. Osterloh JM, et al. dSarm/Sarm1 is required for activation of an injury-induced axon death pathway. Science. 2012;337(6093):481-484.

27. Suzuki M, et al. GDNF secreting human neural progenitor cells protect dying motor neurons, but not their projection to muscle, in a rat model of familial ALS. PLoS ONE. 2007;2(8):e689.

28. Gould TW, et al. Complete dissociation of motor neuron death from motor dysfunction by Bax deletion in a mouse model of ALS. J Neurosci. 2006;26(34):8774-8786. 